\title{
Astrophysical implications of gravitational microlensing of gravitational waves
}

\author{
F. De Paolis, G. Ingrosso, and A. A. Nucita \\ Dipartimento di Fisica, Università di Lecce, and INFN, Sezione di Lecce, Via Arnesano, CP 193, \\ 73100 Lecce, Italy \\ Received 12 October 2000 / Accepted 7 November 2000

\begin{abstract}
Astrophysical implications of gravitational microlensing of gravitational waves emitted by rotating neutron stars (NSs) are investigated. In particular, attention is focused on the following situations: i) NSs in the galactic bulge lensed by a central black hole of $2.610^{6} M_{\odot}$ or by stars and MACHOs distributed in the galactic bulge, disk and halo between Earth and the sources; ii) NSs in globular clusters lensed by a central black hole of $\sim 10^{3} M_{\odot}$ or by stars and MACHOs distributed throughout the Galaxy. The detection of such kind of microlensing events will give a unique opportunity for the unambiguous mapping of the central region of the Galaxy and of globular clusters. In addition, the detection of such events will provide a new test of the General Theory of Relativity. Gravitational microlensing will, moreover, increase the challenge of detecting gravitational waves from NSs.
\end{abstract}

Key words. gravitation - gravitational waves - gravitational lensing

\section{Introduction}

Gravitational microlensing (i.e. gravitational amplification) of electromagnetic waves is a well known phenomenon predicted by the General Theory of Relativity. It is now a well-developed observational technique in astronomy and is considered to be a fundamental tool for acquiring information about the nature and distribution of galactic dark matter.

Indeed, several hundreds of microlensing events have been observed towards the galactic bulge, the Large and Small Magellanic Clouds and even towards the M 31 galaxy (see e.g. Alcock et al. 1997a; De Paolis et al. 1996; Melchior et al. 1998). These observations have led to the conclusion that about $50 \%$ of the galactic dark matter is in the form of MACHOs (Massive Astrophysical Compact Halo Objects) with mass $m \simeq 0.4 M_{\odot}$. Quasar gravitational lensing is a rather sophisticated technique and may even allow investigators, by analyzing the rapid luminosity fluctuations in the lensed images, to establish the nature of the dark matter in the lens galaxies (Lewis \& Belle 1998; Schmidt \& Wambsganss 1998; Mao \& Schneider 1998; Gibson \& Schild 1999).

In the weak field approximation, the physics of gravitational lensing also holds if applied not only to electromagnetic waves (as is usually done) but also to gravitational waves, in particular those emitted by rotating neutron stars (NSs). This may be relevant in relation to

Send offprint requests to: G. Ingrosso the next generation of gravitational wave interferometric detectors, such as VIRGO and LIGO (Jaranowki \& Kròlak 1998). In particular, these detectors will very likely be able to detect gravitational waves emitted by rotating NSs in our galaxy.

In the presence of a massive object (such as a star or a MACHO) close enough to the line of sight of the observed NS, an amplification of the received gravitational wave amplitude $h$ may be observed under certain circumstances. Also, a massive black hole in the center of the Galaxy or in a globular cluster center may induce an amplification of the gravitational waves emitted by NSs situated behind the lensing object. The aim of this paper is to investigate some astrophysical implications of gravitational microlensing of such gravitational waves.

We will concentrate our attention on a few astrophysical situations which appear to be, at first sight, the most interesting: $i$ ) NSs in the galactic bulge lensed by a central black hole with mass $2.610^{6} M_{\odot}$ (hereafter indicated as $\mathrm{BH}$ events) or by stars and MACHOSs distributed throughout the galactic bulge, disk and halo (hereafter indicated as BDH events); ii) NSs in globular clusters lensed by a central black hole of mass $\sim 10^{3} M_{\odot}$ (again BH events) or by stars and MACHOs distributed throughout the Galaxy (BDH events) between Earth and the gravitational wave sources.

In analogy with the standard microlensing analysis (Schneider et al. 1992), when a lens of mass $m$ is sufficiently close to the line of sight of a distant source, 
the gravitational waves emitted by the source suffer a gravitational deflection and the original gravitational wave luminosity increases by the factor

$A=\frac{u^{2}+2}{u\left(u^{2}+4\right)^{1 / 2}}$.

Here $u=d / R_{\mathrm{E}}$ ( $d$ is the distance of the lens from the line of sight to the source) and $R_{\mathrm{E}}$ is the Einstein radius defined as:

$R_{\mathrm{E}}=\left[\frac{4 G m D}{c^{2}} x(1-x)\right]^{1 / 2}$,

where $x=s / D, D$ is the distance between the observer and the source, $s$ is the observer-lens distance. Due to the relative transverse velocity $v_{\perp}$ between the lens and the source (with respect to the line of sight), the amplification factor $A$ is time-dependent and the duration time scale of the microlensing event is given, as usual, by

$\Delta T=\frac{2 R_{\mathrm{E}}}{v_{\perp}}$

Two other quantities are relevant for microlensing and will be used in the following discussion: the optical depth $\tau$, i.e. the fraction of the sky covered by the Einstein circles of all the lenses between the observer and the sources, and the microlensing rate $\Gamma$, which are defined, respectively, as:

$\tau=\int \pi R_{\mathrm{E}}^{2} n(x) \mathrm{d} x$

$\Gamma=2 \iint R_{\mathrm{E}} n(x) v_{\perp} f\left(v_{\perp}\right) \mathrm{d} x \mathrm{~d} v_{\perp}$,

where $n(x)$ is the number density of the considered distribution of lenses and $f\left(v_{\perp}\right)$ is the Maxwellian transverse velocity distribution. For lenses located in the halo or in the bulge of the Galaxy, this function assumes the form $f\left(v_{\perp}\right)=2 v_{\perp} \sigma^{-2} \exp \left(-v_{\perp}^{2} / \sigma^{2}\right)$, where $\sigma$ is the one-dimensional velocity dispersion which turns out to be $210 \mathrm{~km} \mathrm{~s}^{-1}$ and $156 \mathrm{~km} \mathrm{~s}^{-1}$ for halo and bulge objects, respectively (De Rújula et al. 1991). In the case of lenses located in the galactic disk we have to take into account the relative velocity $v_{\mathrm{t}}$ of the observer and the source transverse to the line of sight. The distribution of the transverse velocity for the disk lens is thus $f\left(v_{\perp}\right)=v_{\perp} \sigma^{-2} \exp \left(-\left(v_{\perp}-v_{\mathrm{t}}\right)^{2} / \sigma^{2}\right)$, with $\sigma \simeq$ $30 \mathrm{~km} \mathrm{~s}^{-1}$. In each considered case the integrals on the right hand side of Eqs. (4) and (5) will be performed by taking into account the real geometry of the considered system. The last relevant quantity for a microlensing experiment is the number of events, $N_{\mathrm{ev}}$, detected by observing $N$ neutron stars within an observation time $t_{\mathrm{obs}}$, which is given by

$N_{\mathrm{ev}}= \begin{cases}\Gamma \max \left(\Delta T, t_{\mathrm{obs}}\right) & \text { for BH microlensing } \\ \Gamma N \max \left(\Delta T, t_{\mathrm{obs}}\right) & \text { for BDH microlensing. }\end{cases}$

The matter distributed in our galaxy is assumed to follow a three-component model consisting of a central bulge, a double exponential disk and a dark spherical halo. In particular, the central concentration of stars is described by a triaxial bulge model with a mass density law (Dwek et al. 1995)

$\rho_{\mathrm{C}}(x, y, z)=\frac{M_{\mathrm{b}}}{8 \pi a b c} \mathrm{e}^{-s^{2} / 2}$,

with

$s^{4}=\left(x^{2} / a^{2}+y^{2} / b^{2}\right)^{2}+z^{4} / c^{4}$,

where the bulge mass is $M_{\mathrm{b}} \sim 210^{10} M_{\odot}$ and the scale lengths are $a=1.49 \mathrm{kpc}, b=0.58 \mathrm{kpc}, c=0.40 \mathrm{kpc}$. The coordinates $x$ and $y$ span the galactic disk plane, whereas $z$ is perpendicular to it. The galactic disk is described by a "thin" and a "thick" component (Gilmore et al. 1989). For the "thin" disk we adopt the following density distribution

$\rho_{\mathrm{D}}(R, z)=\frac{\Sigma_{0}}{2 H} \mathrm{e}^{-|z| / H} \mathrm{e}^{-\left(R-R_{0}\right) / h}$,

where the local projected mass density is $\Sigma_{0} \simeq$ $25 M_{\odot} \mathrm{pc}^{-2}$, the scale parameters are $H \simeq 0.30 \mathrm{kpc}$ and $h \simeq 3.5 \mathrm{kpc}$ and $R_{0}=8.5 \mathrm{kpc}$ is the local galactocentric distance. Here $R$ is the galactocentric distance in the plane. For the "thick" component we consider the same density law as in Eq. (9) with thickness $H \simeq 1 \mathrm{kpc}$ and local projected density $\Sigma_{0} \simeq 50 M_{\odot} \mathrm{pc}^{-2}$. We also take into account the effect of the halo dark matter component (in the form of MACHOs) which, as usual, is assumed to follow the mass density profile

$\rho_{\mathrm{H}}(r)=\rho_{0}^{\mathrm{H}} \frac{a^{2}+R_{0}^{2}}{a^{2}+r^{2}}$,

where $\rho_{0}^{\mathrm{H}} \simeq 7.910^{-3} M_{\odot} \mathrm{pc}^{-3}$ is the local dark matter density and $a \simeq 5.6 \mathrm{kpc}$ is the halo core radius. We assume that halo lens objects have a mass $\simeq 0.4 M_{\odot}$ and constitute a fraction $f \simeq 0.5$ of the halo dark matter as shown by microlensing observations (see e.g. Alcock et al. 1997a) ${ }^{1}$.

As far as the mass distribution in globular clusters is concerned, we assume a Plummer density profile given by

$\rho_{\mathrm{GC}}(r)=\rho_{0}^{\mathrm{GC}} \frac{1}{\left[1+\left(r / r_{\mathrm{c}}\right)^{2}\right]^{3 / 2}}$,

where $\rho_{0}^{\mathrm{GC}}$ is the globular cluster central mass density and $r_{\mathrm{c}}$ the core radius.

Our galaxy should contain between a hundred million and a few billions NSs; this follows from an extrapolation of the NS birthrate (Narayan \& Ostriker 1990) and from the number of supernovae required to account for the heavy element abundances in the Milky Way (Arnett et al. 1989). We will assume that the galactic bulge contains about $10^{9}$ NSs. Furthermore, globular clusters are

\footnotetext{
1 Very recently, the MACHO group has reported conclusive results of about 5.7 years of microlensing observations directed towards the LMC. It has been found that the MACHO halo fraction is now $f \simeq 0.2$ for a typical halo model; a fraction $f$ of up to one could still be compatible with observations (Alcock et al. 2000).
} 
expected to contain many NSs which are believed to be a different population with respect to disk NSs. In the following, we will assume that each globular cluster contain $10^{3}$ NSs (Grindlay \& Bailyn 1988).

Rotating NSs are possible astrophysical sources of gravitational waves in the frequency range of interferometric detectors such as VIRGO and LIGO. The emission of gravitational waves, mainly at the neutron star rotation frequency and at twice this frequency (Zimmermann 1980 ), is a consequence of the asymmetric shape of the source caused by internal strong magnetic fields, irregularities in the solid crust or to precessional motion. It is easy to show that the gravitational wave amplitude from a NS at distance $r$ from Earth, rotating with a period $P$, can be expressed as

$$
\begin{aligned}
h_{0} \simeq 4.2 & 10^{-24}\left(\frac{1 \mathrm{~ms}}{P}\right)^{2}\left(\frac{1 \mathrm{kpc}}{r}\right) \\
& \times\left(\frac{I}{10^{45} \mathrm{~g} \mathrm{~cm}^{2}}\right)\left(\frac{\epsilon}{10^{-6}}\right),
\end{aligned}
$$

where $\epsilon$ is the NS ellipticity and $I$ is the moment of inertia. Since highly uncertain, the ellipticity $\epsilon$ is the crucial parameter in this formula, and in order to place limits on the gravitational wave maximum amplitude we shall assume for $\epsilon$ the maximum value obtained by requiring that most of the NS rotational energy is lost by gravitational waves, i.e.

$\epsilon_{\max }=\left(\frac{5 c^{5} P^{3} \dot{P}}{512 \pi^{4} G I}\right)^{1 / 2}$,

where $\dot{P}$ is the rate of deceleration and $I \simeq 10^{45} \mathrm{~g} \mathrm{~cm}^{2}$ is the typical momentum of inertia. For NSs in the galactic bulge we obtain $\epsilon_{\max } \sim 10^{-4}$, since for these objects typical values of $P$ and $\dot{P}$ are $0.1 \mathrm{~s}$ and $10^{-15} \mathrm{~s} \mathrm{~s}^{-1}$, respectively. For globular cluster NSs we obtain $\epsilon_{\max } \sim 10^{-8}$, taking for $P$ and $\dot{P}$ the values $10 \mathrm{~ms}$ and $10^{-18} \mathrm{~s} \mathrm{~s}^{-1}$, respectively (see e.g. Taylor et al. 1993; Camilo et al. 2000).

\section{Microlensing of gravitational waves emitted by rotating NSs}

\subsection{NSs in the galactic bulge}

We first consider NSs located in the galactic bulge microlensed by the central black hole with mass $m \simeq$ $2.610^{6} M_{\odot}$ (Genzel 1998; Ghez et al. 1998). The corresponding Einstein radius is between $R_{\mathrm{E}} \simeq 6-2010^{16} \mathrm{~cm}$, depending on the distance of the source from the galactic center. Assuming that the NS impact parameter is $d \leq R_{\mathrm{E}}$ (implying an amplification $A \geq 1.34$ ), we can use the density distribution law given by Eq. (7) to calculate the rate $\Gamma$ of $\mathrm{BH}$ microlensing events. In this case, Eq. (5) results in $\Gamma \simeq 410^{-3} \mathrm{yr}^{-1} 2$. From Eq. (3), the average

${ }^{2}$ If we also take into account the contribution of the NSs in the galactic disk behind the central black hole, $\Gamma$ increases about $10 \%$. time duration of the NS gravitational wave amplification is $\Delta T \simeq 210^{2} \mathrm{yr}$, which is much longer than the typical integration time for gravitational wave detection given in Table 2 for the VIRGO detector. Such an extended event duration, in addition to giving a substantially enhanced signal, should in principle allow us to construct a Paczyński-like curve (Paczyński 1986) for microlensing of gravitational waves. From Eq. (6), the number $N_{\mathrm{ev}}$ of NSs microlensed by the central black hole is therefore expected to be $\simeq 1$ or higher, possibly up to a few tens, if one takes into account the mass segregation effect and/or a $r^{-7 / 4}$ mass density profile near the galactic center ${ }^{3}$.

Let us consider now BDH microlensing events by stars and MACHOs with an average mass $m \simeq 0.4 M_{\odot}$ distributed throughout the galactic disk, bulge and halo, according to Eqs. $(7-10)^{4}$. In this case, the duration time scale of the gravitational wave amplification event is $\Delta T \simeq 60$ days, about the same value observed for microlensing events of stars towards the galactic bulge (see e.g. Alcock et al. 1997a). Clearly, in this case, even the next generation of gravitational wave detectors will not allow the reconstruction of a Paczyńsky-like curve, but may detect an enhanced gravitational wave amplitude with respect to the non-microlensed NS. From Eqs. (4) and (5) we obtain $\tau \simeq 4.010^{-6}$ and $\Gamma \simeq 2.710^{-5} \mathrm{yr}^{-1}$, in agreement with the estimated optical depth during the first year of optical microlensing observations towards the galactic bulge $^{5}$. We expect there are between $10^{8}$ and $10^{9} \mathrm{NSs}$ in the galactic bulge; therefore, if all these stars could be detected by gravitational wave experiments we would expect to see up to $N_{\mathrm{ev}} \simeq 10^{5} \mathrm{BDH}$ microlensing events within 3 years of integration time. Even if only a minor fraction of NSs could be observed, one would expect to see a few gravitational wave amplification events in a few years of observations.

From Eq. (12) and from the discussion at the end of Sect. 1, the gravitational wave amplitude for bulge NSs is $h_{0} \simeq 310^{-26}$ and, taking into account the microlensing effect, the maximum amplitude of the metric perturbation on Earth is $h_{0}^{\max }=\sqrt{A^{\max }} h_{0}$. It is evident that for impact parameters $d$ much smaller than $R_{\mathrm{E}}$ the amplification parameter $A^{\max }$ increases and, as occurs during the standard microlensing of electromagnetic waves, the gravitational wave amplitude also increases by a factor of 10 or more, thereby facilitating its detection.

\footnotetext{
${ }^{3}$ In particular, taking a $r^{-7 / 4}$ profile up to $r \simeq 10^{-2} \mathrm{pc}$, which corresponds to the position of the last observed star (Genzel 1998; Ghez et al. 1998), we obtain a BH microlensing rate of $\Gamma \simeq 410^{-2} \mathrm{yr}^{-1}$.

${ }^{4}$ We note that towards the galactic bulge, only a negligible number of microlensing events are due to the halo component.

${ }^{5}$ In the first year of the MACHO group observations, 45 events were detected by observing $1.2610^{7}$ stars towards the galactic bulge, with an estimated optical depth $\tau_{b}=$ $3.9_{-1.2}^{+1.8} 10^{-6}$ (Alcock et al. 1997b).
} 
Table 1. Lens Optical depth $\tau$, microlensing rate $\Gamma$ and amplification time scale $\Delta T$ are shown for NSs located in the galactic bulge (GB) and in three globular clusters (NGC 7078, NGC 104 and NGC 6380). The lens location BDH means that lenses are bulge, disk and halo objects. The source location is given in the third, fourth and fifth columns by using galactic coordinates $l$ and $b$ ( $D$ is the source distance from Earth). For each source location, results are given for microlensing by a central black hole (first line) and for microlensing by stars and MACHOs (with assumed mass $m \simeq 0.4 M_{\odot}$ ) distributed throughout the galactic disk, bulge and halo (second line). The $\Gamma$ values for microlensing by the central BH may be considered as lower limits (see text)

\begin{tabular}{lllllllll}
\hline NSs Location & Lens Location & $D(\mathrm{kpc})$ & $l^{\circ}$ & $b^{\circ}$ & Lens Mass $\left(M_{\odot}\right)$ & $\tau$ & $\Gamma\left(\mathrm{yr}^{-1}\right)$ & $\Delta T$ \\
\hline GB & Central BH & 8.5 & 0 & 0 & $2.610^{6}$ & & $4.010^{-3}$ & $210^{2} \mathrm{yr}$ \\
& BDH & 8.5 & & & 0.4 & $4.010^{-6}$ & $2.710^{-5}$ & 60 days \\
\hline NGC 7078 & Central BH & 9.7 & 65.0 & -27.6 & $10^{3}$ & & $3.710^{-7}$ & $8 \mathrm{yr}$ \\
& BDH & 9.7 & & & 0.4 & $1.410^{-7}$ & $9.410^{-7}$ & $60-260$ days \\
\hline NGC 104 & Central BH & 4.6 & 305.89 & -44.88 & $10^{3}$ & $2.510^{-7}$ & $8 \mathrm{yr}$ \\
& BDH & 4.6 & & & 0.4 & $3.910^{-8}$ & $3.510^{-7}$ & $40-200$ days \\
\hline NGC 6380 & Central BH & 4 & 350.18 & -3.414 & $10^{3}$ & & $3.010^{-7}$ & $3 \mathrm{yr}$ \\
& BDH & 4 & & & 0.4 & $1.610^{-7}$ & $4.110^{-7}$ & $40-120$ days \\
\hline
\end{tabular}

Table 2. The gravitational waves amplitude detectable by VIRGO in $3 \mathrm{yr}$ of integration, given as a function of the frequency $f$ (see also Gourgoulhon \& Bonazzola 1996)

\begin{tabular}{|c|c|c|}
\hline$f(\mathrm{~Hz})$ & Sensitivity $\left(\mathrm{Hz}^{-1 / 2}\right)$ & Detectable Amplitude \\
\hline 10 & $10^{-21}$ & $10^{-25}$ \\
30 & $10^{-22}$ & $10^{-26}$ \\
100 & $310^{-23}$ & $310^{-27}$ \\
1000 & $310^{-23}$ & $310^{-27}$ \\
\hline
\end{tabular}

\subsection{NSs in globular clusters}

Observations have shown that in our Galaxy at least 154 globular clusters exist and that each of them contains about $10^{3}$ NSs (Grindlay \& Bailyn 1988). Moreover, about $20 \%$ of the galactic globular cluster population (much more concentrated towards the galactic center) are in a post-core-collapse (PCC) phase and should contain a central massive black hole with mass $\simeq 10^{3} M_{\odot}$ (Chernoff \& Djorgovski 1989). Consequently, gravitational waves from NSs can be microlensed either by the central black hole (BH events) or by lens objects located along the line of sight and distributed through the galaxy (BDH events), according to Eqs. (7-10). In the following, we consider a few PCC globular clusters.

First of all, let us consider the M 15 (NGC 7078) globular cluster with a central black hole of mass $\simeq 10^{3} M_{\odot}$ (Chernoff \& Djorgovski 1989; Gebhardt \& Fisher 1995). We can evaluate the expected number of NSs behind the Einstein circle of the central black hole by assuming they are distributed like the stellar mass density, i.e. following a Plummer density profile. By using Eq. (11) with $r_{\mathrm{c}} \simeq 0.2 \mathrm{pc}$ and $\rho_{0}^{\mathrm{GC}} \simeq 5.110^{6} M_{\odot} \mathrm{pc}^{-3}$, so that the total mass enclosed within about $10 \mathrm{pc}$ is $\simeq 4.810^{5} \mathrm{M}_{\odot}$ (Gebhardt \& Fischer 1995), the obtained BH microlens- ing rate is $\Gamma \simeq 3.710^{-7} \mathrm{yr}^{-1} 6$. However, if we take into account the mass segregation effect and/or a mass distribution law more concentrated towards the center of the PCC globular cluster (with an $r^{-7 / 4}$ profile), the lensing rate may be a factor of 10 higher. In either case, the average amplification time scale is found to be $\Delta T \simeq 8 \mathrm{yr}$.

Let us now consider NSs in M 15 microlensed by lenses in the Galaxy displaced along the line of sight to M 15. By using Eqs. (4) and (5), it is possible to calculate the NS optical depth and the BDH microlensing rate, which turn out to be $\tau \simeq 1.410^{-7}$ and $\Gamma \simeq 9.410^{-7} \mathrm{yr}^{-1}$, respectively. The amplification time scale of the emitted gravitational waves is $\Delta T \simeq 60$ days for MACHOs belonging to the halo population and $\Delta T \simeq 260$ days if the lenses are disk objects.

There are at least two other PCC globular clusters closer to Earth than M 15, and therefore more suitable for this kind of observation: NGC 104 (47 Tucane) and NGC 6380 , at distance of $4.6 \mathrm{kpc}$ and $4 \mathrm{kpc}$, respectively. For the first globular cluster, assuming a Plummer density profile with $r_{\mathrm{c}} \simeq 0.50 \mathrm{pc}$ and $\rho_{0}^{\mathrm{GC}} \simeq 1.110^{5} M_{\odot} \mathrm{pc}^{-3}$, and considering gravitational waves from NSs microlensed by the central black hole, we get a $\mathrm{BH}$ event rate $\Gamma \simeq$ $2.510^{-7} \mathrm{yr}^{-1}$ and an amplification time scale $\Delta T \simeq 8 \mathrm{yr}$. For the microlensing events due to stars and/or MACHOs distributed throughout the Galaxy, we get $\tau \simeq 3.910^{-8}$ and $\Gamma \simeq 3.510^{-7} \mathrm{yr}^{-1}$, while the average event duration is $\Delta T \simeq 40$ days for MACHOs in the galactic halo and $\Delta T \simeq 200$ days for lenses in the galactic disk.

Finally, we considered the PCC globular cluster NGC 6380 described with a Plummer model with $r_{\mathrm{c}} \simeq$ $0.40 \mathrm{pc}$ and $\rho_{0}^{\mathrm{GC}} \simeq 4.410^{4} M_{\odot} \mathrm{pc}^{-3}$. In the case of $\mathrm{BH}$ microlensing events we get $\Gamma \simeq 310^{-7} \mathrm{yr}^{-1}$,

\footnotetext{
${ }^{6}$ If we consider a King model for M 15 with tidal radius $r_{\mathrm{t}}=1.3 \mathrm{pc}$, core radius $r_{\mathrm{c}}=0.1 \mathrm{pc}$, central density $\rho_{0}^{\mathrm{GC}}=$ $4.210^{6} M_{\odot} \mathrm{pc}^{-3}$ and of the same total mass (Gebhardt \& Fisher 1995; Lehmann \& Scholz 1997) instead of a Plummer model, $\Gamma$ does not change substantially.
} 
corresponding to an average amplification time scale $\Delta T \simeq 3$ yr. For $\mathrm{BDH}$ microlensing events we get in this case $\tau \simeq 1.610^{-7}$ and $\Gamma \simeq 4.110^{-7} \mathrm{yr}^{-1}$, while the event duration is $\Delta T \simeq 40-120$ days. These results are summarized in Table 1 .

\section{Discussion and conclusions}

In this paper, we have discussed the astrophysical implications of gravitational microlensing of gravitational waves emitted by rotating NSs located in the galactic bulge and in a few globular clusters. We consider this subject because with the next generation of gravitational wave interferometric detectors, it should be possible to obtain information on the galactic NS population by studying emitted gravitational waves. As shown in Eq. (12), the amplitude of these waves can be expressed in terms of the NS rotation period $P$, distance $r$ to Earth, moment of inertia $I$ and ellipticity $\epsilon$ which we estimate by Eq. (13). With the assumptions we made for $P, \dot{P}$ and $\epsilon_{\max }$, the amplitude of the gravitational waves emitted by NSs located in the galactic bulge and in globular clusters can be expressed, respectively, as

$h_{0}^{\text {Bulge }} \simeq 2.510^{-25}\left(\frac{\mathrm{kpc}}{r}\right), h_{0}^{\mathrm{GC}} \simeq 7.910^{-26}\left(\frac{\mathrm{kpc}}{r}\right)$.

The detectability of gravitational waves from NSs by VIRGO-like detectors was studied by many authors (see e.g. Shutz 1991; Gourgoulhon \& Bonazzola 1996). These authors pointed out that any pulsar (with $P \simeq 0.1 \mathrm{~s}$ ) emitting gravitational waves with amplitude $h_{0}$ greater than $10^{-26}$ in the frequency bandwidth where the sensitivity of the detector is better than $10^{-22} \mathrm{~Hz}^{-1 / 2}$, can be detected in 3 years of integration time (see Table 2). With this threshold, it is possible to detect the gravitational waves emitted by rotating NSs within about $25 \mathrm{kpc}$, using the VIRGO detector. Clearly, when we take into account the gravitational microlensing effect, the amplitude of the gravitational waves is increased, thereby facilitating their detection. As is shown by standard microlensing observations towards the galactic bulge, the amplification factor $A^{\max }$ may be very high. In fact, there are events with $A^{\max } \simeq 70$ (Alcock et al. 1997b; MACHO Project Home Page 2000), although the mean amplification averaged since 1993 to 1999 is $A^{\max } \simeq 4$.

It is also easy to understand that the detection and the study of the gravitational waves emitted from NSs can give remarkable information regarding the population of pulsars inside the Galaxy and globular clusters, providing a way to test current evolutionary theories. Indeed, with the present astronomical observation techniques it is not possible to distinguish objects behind the massive central black holes and/or in the inner regions of the galactic and globular cluster centers. However, gravitational wave detectors offer, in principle, a powerful tool for this kind of astronomical investigation. If the gravitational wave signal is higher than the background noise or if it is sufficiently enhanced, for example by the microlensing effect, we should be able to observe NSs which cannot be seen by any other observational method. It is also true that the present generation of interferometric detectors lacks sufficiently high angular resolution to distinguish, as different gravitational waves sources, NSs which are very close to each other. For example, Jaranowki \& Kròlak (1998) showed that VIRGO is expected to have an angular resolution $\Delta \Omega \simeq 5.610^{-8} \mathrm{sr}$ (corresponding to an angular separation $\Delta \theta \simeq 27.5^{\prime \prime}$ ), i.e. in the center of our Galaxy, two NSs, with a separation distance 1 pc, should be observed as different sources. Otherwise, advanced LIGO detectors should have a better angular resolution $\left(\Delta \Omega \simeq 7.910^{-10}\right.$ sr corresponding to $\left.\Delta \theta \simeq 3.3^{\prime \prime}\right)$, so that NSs 0.1 pc apart could be resolved. The next generation of interferometric detectors, combined with a network analysis of the gravitational wave signal, will likely increase the angular resolution which can be obtained. In general, two images are seen as two resolved sources if the angular resolution of the telescope is below their angular separation, $\Delta \theta_{1}$, whose expression is

$\Delta \theta_{\mathrm{l}} \simeq 2 \frac{R_{\mathrm{E}}}{s} \sqrt{u^{2}+4}$.

In the case of the black hole at the galactic center, the previous equation results in $\Delta \theta_{1} \simeq 2.1^{\prime \prime}$, taking a NS at a distance of $1 \mathrm{kpc}$ from the central black hole. Two images separated by $2.1^{\prime \prime}$ should be resolvable, for example, by optical telescopes, however leaving open the problem of detecting the lensing effect despite the large number of background stars. In the case of gravitational wave detectors, the lensed object is seen as a gravitational microlensing event (i.e. gravitational wave amplification) due to the more rough angular resolution. In the case of the globular cluster NGC 6380, the angular separation turns out to be $\Delta \theta_{1} \simeq 910^{-3^{\prime \prime}}$. Therefore, a lensed source is seen as an amplitude amplification (i.e. as a microlensing event).

We also note that recently Ruffa (1999) studied the gravitational lensing of gravitational waves from a completely different point of view. He considered gravitational wave amplification as a consequence of the Fraunhofer diffraction by a ring of radius $R_{\mathrm{E}}+\Delta R_{\mathrm{E}}$ centered at the lens. In the case of gravitational waves from NSs amplified by the black hole at the galactic center he found an expected amplification $A=1.710^{4}$, which could significantly aid observations. The problem with this analysis is the requirement for the perfect alignment of the lens and the source with the observer, which should be an extremely unlikely situation in astrophysics. In a following paper (De Paolis et al. 2000), Ruffa's treatment is generalized to the situation of a non-aligned system in which the lens pass at some impact parameter with respect to the line of sight to the source.

We emphasize that detecting such kind of microlensing events, in addition to giving us a new test of the General Theory of Relativity, should allow us to increase our knowledge of the galactic center region and to constrain the NS distribution in the galactic bulge, since 
the only possibility of detecting NSs behind the central black hole is through the emitted gravitational waves. Such gravitational events provide information about the nature of the lensing object, allowing us, in principle, to discriminate between a massive black hole and, for example, a compact star cluster (Maoz 1998) or a neutrino star (Munyaneza et al. 1998; Capozziello \& Iovane 1999) at the galactic center.

Detecting microlensing events of NSs in globular clusters is also important in order to obtain information about the evolution of a globular cluster, i.e. about the PCC phase and/or the mass segregation effect.

\section{References}

Alcock, C., Allsman, R. A., Alves, D., et al. 1997a, ApJ, 486, 697

Alcock, C., Allsman, R. A., Alves, D., et al. 1997b, ApJ, 479, 119

Alcock, C., Allsman, R. A., Alves, D., et al. 2000, ApJ, 542, 281

Arnett, W. D., Schramm, D. N., \& Truran, J. W. 1989, ApJ, 339, L25

Binney, J., \& Tremaine, S. 1987, Galactic Dynamics (Princeton: Princeton University Press)

Brady, P. R., Creighton, T., Cutler, C., \& Schutz, B. F. 1998, Phys. Rev. D, 57, 4

Camilo, F., Lorimer, D. R., Freire, P., et al. 2000, ApJ, 535, 975

Capozziello, S., \& Iovane, G. 1999, Phys. Lett. A, 259, 185

Chernoff, D. F., \& Djorgovski, S. 1989, ApJ, 339, 904

De Paolis, F., Ingrosso, G., \& Jetzer, Ph. 1996, ApJ, 470, 493

De Paolis, F., Ingrosso, G., \& Nucita, A. A. 2000, in preparation
De Rújula, A., Jetzer, Ph., \& Massò, E. 1991, MNRAS, 250, 348

Dwek, E., Arendt, R. G., Hauser, M. G., et al. 1995, ApJ, 445, 716

Gebhardt, K., \& Fisher, Ph. 1995, ApJ, 109, 209

Genzel, R. 1998, Nature, 391, 42

Ghez, A. M., Klein, B. L., Morris, M., \& Becklin, E. E. 1998, ApJ, 509, 678

Gibson, \& Schild, R. 1999, preprint [astro-ph/9904362]

Gilmore, G., Wyse, R. F. G., \& Kuijken, K. 1989, ARA\&A, 27,555

Gourgoulhon, E., \& Bonazzola, S. 1996, Proceedings of the International Conference on Gravitational Waves: Sources and Detectors, Italy: World Scientific

Grindlay, J. E., \& Bailyn, C. D. 1988, Nature, 336, 48

Jaranowski, P., \& Kròlak, A. 1998, pre-print [gr-qc/9809046]

Lehmann, I., \& Scholz, R.-D. 1997, A\&A, 320, 776

Lewis, G. F., \& Belle, K. E. 1998, MNRAS, 297,69

Mao, S., \& Schneider, P. 1998, MNRAS, 295, 587

Maoz, E. 1998, ApJ, 494, L181

Melchior, A. L., Afonso, C., Ansari, R., et al. 1998, A\&A, 339, 658

Munyaneza, F., Tsiklauri, D., \& Viollier, R. D. 1998, ApJ, 509, L105

Narayan, R., \& Ostriker, J. O. 1990, ApJ, 352, 222

Paczyński, B. 1986, ApJ, 304, 1

Ruffa, A. A. 1999, ApJ, 517, L31

Schmidt, R., \& Wambsganss, J. 1998, A\&A, 335, 379

Schutz, B. F. 1991, The Detection of Gravitational Waves (Cambridge: Cambridge university Press)

Schneider, P., Ehlers, J., \& Falco, E. E. 1992, Gravitational Lenses (Cambridge: Springer-Verlag)

Taylor, J. H., Manchester, R. N., \& Lyne, A. G. 1993, ApJS, 88,529

Zimmermann, M. 1980, Phys. Rev. D, 21, 4

MACHO project home page at http://darkstar.astro. washington.edu 